\title{
Factors Influencing the Mantel-Haenszel Procedure in the Detection of Differential Item Functioning
}

\author{
Thomas Uttaro, Rutgers University \\ Roger E. Millsap, Baruch College, CUNY
}

The performance of the Mantel-Haenszel oddsratio estimator and $\chi^{2}$ significance test were investigated using simulated data. Multiparameter logistic item response theory models were used to generate item scores for 20 -and 40 -item tests for 500 reference group and 500 focal group examinees. The difficulty, discrimination, and guessing parameters, and the difference in the group trait level averages were varied and combined factorial ly. Within each cell of the design, 200 replications were completed under both differential item functioning (DIF) and no-DIF conditions. The empirical $\chi^{2}$ Type $\mathbb{I}$ and Type II error rates, and the average of the odds-ratio estimates, were analyzed over the 200 replications. Under no-DIF conditions, inflated $x^{2}$ Type 1 error rates and misestimated odds-ratio values were found for the 20-item test and resulted from interactions between item parameter values and trait differences. For the 40 -item test, Type $\mathbb{I}$ error rate inflation disappeared but odds-ratios still were misestimated. Under DIF conditions, Type II error rates were not inflited, but oddsratios were misestimated, due to parameter $\times$ trait level interactions for both test lengths. The results demonstrate the importance of using both the oddsratio and the significance test in interpreting the presence or absence of DIF. In addition, the accuracy under the DIF conditions depended on the size and uniformity of DIF. Index terms: differential item functioning, item bias, item response theory, Mantel-Haenszel procedure, measurement bias, oddsratio, simulation.

The Mantel-Haenszel (MH) procedure (Mantel \& Haenszel, 1959) is a simple and inexpensive differential item functioning (DIF) detection technique that is used widely (Holland \& Thayer, 1988). The accuracy of the procedure in detect-

APPLIED PSYCHOLOGICAL MEASUREMENT

Wol. 18, No. 1, March 1994, pp. 15-25

(c) Copyright 1994 Applied Psychological Measurement Inc. 0146-6217/94/010015-11\$1.80 ing DIF has not been thoroughly investigated. The present study used simulated data to investigate whether the $M H$ procedure detects DIF with adequate power under realistic conditions and whether it holds the Type I error rate to an acceptable level.

\section{The MH Procedure}

The MH procedure for DIF detection stratifies the reference and focal examinee groups according to measured trait level. The item performance of the focal group (e.g., females) is of primary interest and is compared to that of the reference group (e.g., males), after matching on the measured trait. The total test score usually serves as the estimate of trait level on which examinees are matched. Within each group of examinees with the same total test score, the pass/fail frequencies on the studied item are used for both the reference and focal groups to create $22 \times 2$ contingency table. If there are $K$ matched groups, there will be $K 2 \times 2$ tables. The MH procedure tests the null hypothesis that the common oddsratio, $a_{\mathrm{Mm}}$ across the $K$ tables is equal to 1.0 . The MH odds-ratio estimator is

$\hat{\alpha}_{\mathrm{MH}}=\frac{\sum_{k} A_{k} D_{k} / T_{k}}{\sum_{k} B_{k} C_{k} / T_{k}}$,

where, in $\mathbb{K} 2 \times 2$ tables,

$A_{k}$ and $\mathbb{C}_{k}$ are the numbers of examinees in the reference and focal groups, respectively, who answer the item correctly,

$D_{k}$ and $B_{k}$ are the numbers of examinees in the reference and focal groups, re- 
spectively, who answer the item incorrectly, and

$T_{k}$ is the total number of reference and focal group examinees in score level $k$.

A test statistic, $\chi_{\mathrm{MH}}^{2}$, provides a $\chi^{2}$ test of this hypothesis. The $\chi^{2}$ significance test of the oddsratio estimator (with continuity correction) is

$$
\chi_{\mathrm{MH}}^{2}=\frac{\left[\left|\sum_{k} A_{k}-\sum_{k} \mathrm{E}\left(A_{k}\right)\right|-\frac{1}{2}\right]^{2}}{\sum_{k} \operatorname{Var}\left(A_{k}\right)},
$$

where $\mathbb{E}\left(A_{k}\right)$ and $\operatorname{Var}\left(A_{k}\right)$ are the expected value and variance of $A$ at score level $k$. Further computational details may be found in Holland \& Thayer (1988) or Donoghue, Holland, \& Thayer (1993). Rejection of the unit $\alpha_{M H}$ hypothesis suggests that DIF is present in the studied item. The estimate of $\alpha_{M H}$ is a useful descriptive index of DIF.

\section{Relationship Whith DEF Rrocedures Based on Itern Response Theory}

The $\alpha_{\mathrm{MH}}$ hypothesis is related to model-based definitions of DIF under certain conditions. Using item response theory (IRT), Lord (1980) defined DIF as group differences in item response functions (IRFs). Holland \& Thayer (1988) noted that the MH and IRT definitions of DIF are equivalent if

1. All items including the studied item follow the Rasch model,

2. There is no DIF in items other than the studied item,

3. The matching criterion includes the studied item, and

4. The data are random samples of the focal and reference groups.

Under these conditions, the total test score is a sufficient statistic for the unknown trait level parameter $\theta$ (Lewis, 1993; Meredith \& Millsap, 1992).

It is not clear how the procedure performs under more general conditions, when sufficiency is violated. Several theoretical studies have examined this question. Zwick (1990) studied the equivalence of the IRT and MH definitions of DIF within a broad class of items with strictly monotone IRFS and local independence. This class includes items following the logistic IRT models. In the twoparameter logistic IRT model (2PLM), the two definitions were found to be equivalent only if the $\theta$ distributions in the reference and focal groups were identical, or if the discrimination parameter values were identical for all items (implying a Rasch model). Stochastic ordering of the $\theta$ distributions was shown to lead to rejection of the MH null hypothesis when no DIF was present.

Meredith \& Millsap (1992; Millsap \& Meredith, 1992) studied measurement bias detection methods that match examinees on observed scores, such as the MH procedure. They demonstrated the importance of sufficiency of the observed matching variable for trait level in determining the performance of these methods. If the matching variable is itself unbiased and is a sufficient statistic for the latent trait, invariance of IRFs implies the unit $\alpha_{\mathrm{MH}}$ hypothesis. When examinees are matched on total test score, near sufficiency is achieved in long tests under very general conditions.

Collectively, these theoretical studies suggest that the unit $\alpha_{M H}$ hypothesis and IRF invariance are strictly equivalent only under special conditions. In short tests, such as those with 20 or fewer items, or when the items do not fit the Rasch model, the equivalence breaks down. Questions about the accuracy of the $\mathrm{MH}$ procedure in these cases may be answered using simulations.

\section{Sinnulation Studies}

Several studies have examined the accuracy of the $\mathrm{MH}$ procedure in data simulated to represent DIF or the absence of DIF. Typically, item scores are generated under a specific IRT model with varying degrees of DIF. Other influences on detection accuracy, such as test length or the inclusion of the studied item in the matching variable, also may be varied.

Donoghue et al. (1993) conducted a large simulation study varying several factors believed to influence detection accuracy. Item scores were generated under the three-parameter logistic model (3PLM),

$P_{i}(\theta)=c_{i}+\left(1-c_{i}\right)\left\{1+\exp \left[-D a_{i}\left(\theta-b_{i}\right)\right]\right\}^{-1}$, 
where

$a$ is the item discrimination parameter,

$b$ is the item difficulty parameter, and

$c$ is the item pseudo-guessing parameter.

In Donoghue et al. (1993), $c$ was set to a constant. DIF was represented by group differences in $b$

only. $a$ values were identical across items within a given cell of the design, but this common value was varied systematically. The dependent measure of interest was the value of the estimated $\alpha_{\mathrm{MH}}$, rescaled to the ETS difficulty metric.

Three factors had strong main effects on the rescaled $\alpha_{\mathrm{MH}}$ : inclusion of the studied item in the matching variable, the $b$ value of the studied item, and the amount of DIF in the studied item. Inclusion of the studied item in the matching variable was found to be necessary for accurate detection, confirming theoretical analyses (Holland \& Thayer, 1988; Meredith \& Millsap, 1992; Zwick, 1990). The amount of DIF in the studied item was positively related to the power of the procedure but was moderated by the sizes of the $a$ and $b$ parameters. Even though they were varied in a restricted manner, the study suggested that item parameter values can influence $\mathrm{MH}$ results in complex ways.

In a second simulation study, Swaminathan \& Rogers (1990; Rogers \& Swaminathan, 1990) compared the MH procedure to a logistic regression procedure. The $\mathrm{MH}$ dependent variable of interest was the power of the procedures to detect DIF as estimated by the empirical proportion of correct positives identified through $\chi_{M H}^{2}$ across replications. This study focused on the performance of the two methods under "uniform" and "nonuniform" DIF conditions. Under uniform DIF, the group difference in the IRFs has the same sign throughout the trait range, with the advantage always given to one group. Under nonuniform DIF, the group difference reverses in sign at some point in the trait range. Data were generated under the 3 PLM with fixed $c$ values. Uniform DIF was created by introducing group differences in the $b$ s only. Nonuniform DIF was created by introducing group differences in as, but using identical $b$ values, therefore producing symmetric nonuni- form DIF about the intersection point of the modeled IRFs. Other factors varied in the study included sample size, test length, amount of DIF in the studied item, shape of the test score distribution, and proportion of biased items in the test.

The MH procedure was found to have generally adequate power for detecting uniform DIF, but low power for detecting (symmetric) nonuniform DIF. Power was higher in larger samples (250 vs. 500) and for larger amounts of DIF. In the nonuniform conditions, power varied with the size of the $b$ parameter in the studied item; power was weakest for items with medium $b$ values (i.e., $-.6 \leq b \leq .6)$. Test length was not found to be a significant influence, but only relatively long tests were used (40-80 items).

Although these simulation studies produced important findings, each had limitations. Donoghue et al. (1993) only examined the uniform DIF case for the rescaled $\alpha_{\mathrm{MH}}$, and did not evaluate the performance of $\chi_{\mathrm{MH}}^{2}$. Furthermore, all items within a given condition had identical $a$ and $c$ parameter values. This item homogeneity is unlikely in real data. Rogers \& Swaminathan (1990) studied both uniform and nonuniform DIF, but did not consider short tests (less than 40 items). They evaluated the performance of $\chi_{M H}^{2}$ but not the $\alpha_{\mathrm{MH}}$. The classes of uniform and nonuniform DIF modeled were rather restricted, and $a$ and $b$ values were not simultaneously varied in the uniform or nonuniform conditions. Neither study examined the influence of group differences in the $c$ parameters under the 3PLM.

\section{Prapose}

The present study addressed these limitations. Both uniform and nonuniform DIF conditions were simulated, and parameter values within the 3PLM were varied systematically. Group differences in $c$ parameters were present in some conditions. The $a$ parameters for items in the matching criterion were varied, departing from the Rasch model case. Both short (20 items) and moderate ( 40 items) test lengths were used. Results are presented for both $\chi_{\mathrm{MH}}^{2}$ and $\alpha_{\mathrm{MH}}$ under identical conditions. No previous study has exam- 
ined both these statistics under the broad class of conditions incorporated here.

\section{Method}

Simulated datasets were generated from logistic IRFs. IRF parameters $(a, b$, and $c)$ for each item were input for the one-parameter logistic model, the 2PLM, and the 3PLM; $\theta$ distributions for the reference and focal groups were $\mathrm{N}(0,1)$ and $\mathrm{N}(0,1)$, respectively, for the no-discrepancy condition and $\mathrm{N}(0,1)$ and $\mathrm{N}(-1,1)$, respectively, for the discrepant condition. In the no-discrepancy condition, measured trait distributions for both groups were centered at 0.0 . In the discrepant condition, the mean measured trait for the focal group was $\mathbb{1}$ standard deviation below the reference group mean of 0.0 . The program produced scaled $\theta$ values for each examinee, and the probability of a correct response was given by Equation 3 .

Because $\theta$ and the item parameter values were known, $P_{i}(\theta)$ was determined. The item score was generated when the program selected a random number from a uniform distribution $(0,1)$. If the random number was less than or equal to $P_{i}(\theta)$, the examinee received a score of 1 , otherwise the examinee received a score of 0 . This process was repeated for each item and examinee.

For each phase of the study the matching criterion was total score, including the studied item, with no bias in items other than the studied item. The set of IRF parameters and $\theta$ levels for the studied item were determined using a $3 \times 3 \times 2 \times 2$ design. The independent variables (factors) were:

1. A, 3 levels of $a: .5,1.0,1.5$;

2. B, 3 levels of $b: 0.0, .3, .5$;

3. $\mathrm{C}, 2$ levels of $c: 0.0, .2$; and

4. $\theta_{\text {dis }}$, the reference and focal group discrepancy in mean $\theta$ level. There were 2 levels of $\theta_{\text {dis }}$ : $0.0,-1.0$.

Each combination of these factors produced DIF and no-DIF conditions. For the no-DIF conditions, the IRFS were identical for the reference and focall groups with parameter values equal to the cell factor values. For the DIF conditions, the focal group IRFS were defined by the cell factor values, and the reference group IRF parameters were set at $a=1.0, b=0.0$, and $c=.2$.

The IRF parameterizations of matching items, other than the studied item, were combinations of a parameter values $(.5,1.0,1.5)$ representing low, moderate, and high discrimination; $b$ parameter values $(-2.25$ to 2.25$)$ covering a wide range of difficulty values; and $c$ parameter values $(0, .1, .2)$. Table 1 shows the 36 cases that were defined in each cell of the design. Note, for example, that Cases 1-6 have the same levels of $a, b$, and $c$ as Cases 19-24 but these two groups of cases differ on $\theta_{\text {dis }}$. There were 500 examinees in both the reference and focal groups, and data were analyzed for both 20 -item and 40 -item tesis.

Each cell in the above factorial design was replicated 200 times, under both no-DIF and DIF conditions. In each replication, new item scores for the 500 examinees in each group were generated as described above. Once the item scores were generated, both $\chi_{\mathrm{MH}}^{2}$ and $\alpha_{\mathrm{MH}}$ were calculated for the studied item. The average value of $\alpha_{M H}$ across the 200 replications was computed. In the no-DIF conditions, the number of replications in which the $\chi_{\text {мн }}^{2}$ exceeded the critical value at an $\alpha$ level of .05 was recorded. This was the empirical Type I error rate. In the DIF conditions, the number of replications in which $\chi_{\mathrm{MH}}^{2}$ failed to exceed this critical value was recorded. This was the empirical Type II error rate.

ANOVA was used to test for significant factors under the no-DIF condition using the $\chi_{\mathrm{MH}}^{2}$ Type I error rate and $\alpha_{\mathrm{MH}}$ as dependent variables. The $A, B$, and $C$ parameter factors and the $\theta_{\text {dis }}$ trait level factor were the independent variables. For the DIF condition, the form and degree of DIF (i.e., uniform or nonuniform DIF) required different analyses than the no-DIF condition. In the DIF condition, ANOVAs were conducted for the focal group variables based on the form and degree of DIF. The form and degree of DIF were analyzed using closed signed area (CSA) and closed unsigned area (CUA) measures between IRFs, bounded on the interval $(-3 \leq \theta \leq 3)$, based on equations provided by Kim \& Cohen (1991). 
Table 1

$\theta_{\text {dis }}, a, b$, and $c$ Values by Case Number;

Parameter Values Are for Both the Reference and Focal Groups in No-DIF Conditions; for the DIF Conditions, Parameter Values Are for the Focal Group and Reference Group

Parameters $\mathbb{W}$ ere $a=1.0, b=0.0$, and $c=.2$

\begin{tabular}{crrrr}
\hline Case & & & & \\
Number & $\theta_{\text {dis }}$ & $a$ & \multicolumn{1}{c}{$b$} & \multicolumn{1}{c}{$c$} \\
\hline 1 & 0.0 & .5 & 0.0 & 0.0 \\
2 & 0.0 & .5 & .3 & 0.0 \\
3 & 0.0 & .5 & .5 & 0.0 \\
4 & 0.0 & .5 & 0.0 & .2 \\
5 & 0.0 & .5 & .3 & .2 \\
6 & 0.0 & .5 & .5 & .2 \\
7 & 0.0 & 1.0 & 0.0 & 0.0 \\
8 & 0.0 & 1.0 & .3 & 0.0 \\
9 & 0.0 & 1.0 & .5 & 0.0 \\
10 & 0.0 & 1.0 & 0.0 & .2 \\
11 & 0.0 & 1.0 & .3 & .2 \\
12 & 0.0 & 1.0 & .5 & .2 \\
13 & 0.0 & 1.5 & 0.0 & 0.0 \\
14 & 0.0 & 1.5 & .3 & 0.0 \\
15 & 0.0 & 1.5 & .5 & 0.0 \\
16 & 0.0 & 1.5 & 0.0 & .2 \\
17 & 0.0 & 1.5 & .3 & .2 \\
18 & 0.0 & 1.5 & .5 & .2 \\
19 & -1.0 & .5 & 0.0 & 0.0 \\
20 & -1.0 & .5 & .3 & 0.0 \\
21 & -1.0 & .5 & .5 & 0.0 \\
22 & -1.0 & .5 & 0.0 & .2 \\
23 & -1.0 & .5 & .3 & .2 \\
24 & -1.0 & .5 & .5 & .2 \\
25 & -1.0 & 1.0 & 0.0 & 0.0 \\
26 & -1.0 & 1.0 & .3 & 0.0 \\
27 & -1.0 & 1.0 & .5 & 0.0 \\
28 & -1.0 & 1.0 & 0.0 & .2 \\
29 & -1.0 & 1.0 & .3 & .2 \\
30 & -1.0 & 1.0 & .5 & .2 \\
31 & -1.0 & 1.5 & 0.0 & 0.0 \\
32 & -1.0 & 1.5 & .3 & 0.0 \\
33 & -1.0 & 1.5 & .5 & 0.0 \\
34 & -1.0 & 1.5 & 0.0 & .2 \\
35 & -1.0 & 1.5 & .3 & .2 \\
36 & -1.0 & 1.5 & .5 & .2 \\
\hline & & & &
\end{tabular}

ResuIts

Mean values of $\chi_{\mathrm{MH}}^{2}$ error rates and $\alpha_{\mathrm{MH}}$ across the 200 replications for each case are presented in Table 2. The significant effects for the entire set of ANOVAs, including the nO-DIF and DIF conditions, are summarized in Table 3.

\section{No-D正 Condition}

20-item test. ANOVAs for $\chi_{\mathrm{MH}}^{2}$ Type I error rates yielded significance for the $\theta_{\text {dis }}$ main effect, the $\mathrm{A} \times \mathbb{C}$ interaction, and the $\mathbb{A} \times \mathbb{C} \times \theta_{\text {dis }}$ interaction (see Table 3). The $\mathbb{A} \times \mathbb{C} \times \theta_{\text {dis }}$ interaction for $\theta_{\text {dis }}=-1.0$ (means are given in Table 4) is of considerable practical and statistical significance, because 20-item tests (or subtests) using the 3PLM with measured trait discrepancies are not uncommon. Type I error rate levels were higher than the 05 level for $\theta_{\mathrm{dis}}=-1.0$, whereas for $\theta_{\mathrm{dis}}=0.0$ all Type I error rates were below .05. The conditions that deviated most sharply from the .05 significance level were $\theta_{\mathrm{dis}}=-1.0, a=.5$, and $c=.2(.12)$ and $\theta_{\text {dis }}=-1.0, a=1.5$, and $c=0.0(.16)$.

Similar results were obtained for $\alpha_{\mathrm{MH}}$. Significance was obtained for the $A, C$, and $\theta_{\text {dis }}$ main effects, and the $\mathrm{A} \times \mathbb{C}, \mathrm{A} \times \theta_{\text {dis }}, \mathrm{C} \times$ $\theta_{\text {diss }}$ and $\mathbf{A} \times \mathbb{C} \times \theta_{\text {dis }}$ interactions (Table 3 ). Means for the $\mathrm{A} \times \mathrm{C} \times \theta_{\text {dis }}$ interaction also are presented in Table 4 .

All of the $\alpha_{\mathrm{MH}}$ values corresponding to $\theta_{\text {dis }}=0.0$ were quite close to 1.0 , accurately reflecting the no-DIF condition. However, for $\theta_{\mathrm{dis}}=-1.0$ the $\alpha_{\mathrm{MH}}$ values deviated considerably from 1.0. As in the $\chi_{M H}^{2}$ analyses above, the cells that deviated most sharply were $\theta_{\text {dis }}=-1.0$, $a=.5$, and $c=.2(.89 ;$ Cases $22-24$ in Table 1) and $\theta_{\mathrm{dis}}=-1.0, a=1.5$, and $c=0.0$ (1.30; Cases $31-33)$. Table 2 shows the corresponding cases for the same values of $\theta, a$, and $c$ at all levels of $b$ (e.g., for Case 22 the $\chi_{M H}^{2}$ was .13 and the $\alpha_{\mathrm{MH}}$ was .90 , and for Case 31 the $\chi_{\mathrm{MH}}^{2}$ was .19 and the $\alpha_{\mathrm{MH}}$ was 1.29 ).

40-item test. The ANOVA for the $\chi_{\mathrm{MH}}^{2}$ Type I error rates produced no significant effects, although the $A$ main effect approached significance $(F=6.77, p<.06)$. The mean Type 1 error rates for the three levels of $\mathbb{A}$ were .06 , .05 , and .05 . The ANOVA for $\alpha_{M H}$ provided a significant $\mathrm{C}$ main effect and significant $\mathrm{A} \times$ $\theta_{\mathrm{dis}}$ and $\mathbb{C} \times \theta_{\mathrm{dis}}$ interactions. Means for the $\mathrm{A} \times \theta_{\text {dis }}$ and $\mathrm{C} \times \theta_{\text {dis }}$ interactions are shown in Table 5 . 
Table 2

Mean $\chi_{M H}^{2}$ Type I Error Rates (No-DIF Condition), Mean $\chi_{\mathrm{MH}}^{2}$ Type II Error Rates (DIF Condition) and Mean $\alpha_{\mathrm{MH}}$ for the 20-Item and 40-Item Tests.

\begin{tabular}{|c|c|c|c|c|c|c|c|c|}
\hline \multirow{3}{*}{$\begin{array}{l}\text { Case } \\
\text { Number } \\
\end{array}$} & \multicolumn{4}{|c|}{ Type I Error Rates } & \multicolumn{4}{|c|}{ Type II Error Rates } \\
\hline & \multicolumn{2}{|c|}{ 20-Item Test } & \multicolumn{2}{|c|}{ 40-Item Test } & \multicolumn{2}{|c|}{ 20-Item Test } & \multicolumn{2}{|c|}{ 40-Item Test } \\
\hline & $\chi_{\mathrm{MH}}^{2}$ & $\alpha_{\mathrm{MH}}$ & $\chi_{M H}^{2}$ & $\alpha_{\mathrm{MH}}$ & $\chi_{\mathrm{MH}}^{2}$ & $\alpha_{\mathrm{MH}}$ & $\chi_{\mathrm{MH}}^{2}$ & $\alpha_{\mathrm{MH}}$ \\
\hline 1 & .05 & 1.02 & .07 & 1.02 & .08 & 1.66 & .07 & 1.69 \\
\hline 2 & .04 & 1.02 & .07 & 1.01 & 0.00 & 2.22 & 0.00 & 2.21 \\
\hline 3 & .03 & 1.02 & .05 & 1.01 & 0.00 & 2.64 & 0.00 & 2.65 \\
\hline 4 & .03 & 1.00 & .06 & 1.01 & .96 & 1.01 & .96 & 1.03 \\
\hline 5 & .05 & 1.00 & .05 & 1.02 & .71 & 1.26 & .66 & 1.27 \\
\hline 6 & .05 & 1.01 & .08 & 1.02 & .37 & 1.42 & .30 & 1.45 \\
\hline 7 & .05 & 1.04 & .05 & 1.02 & .09 & 1.80 & .03 & 1.80 \\
\hline 8 & .04 & 1.01 & .04 & 1.02 & 0.00 & 2.83 & 0.00 & 2.90 \\
\hline 9 & .03 & 1.01 & .05 & 1.02 & 0.00 & 3.92 & 0.00 & 4.05 \\
\hline 10 & .03 & 1.01 & .04 & 1.03 & - & - & - & - \\
\hline 11 & .06 & 1.02 & .05 & 1.02 & .39 & 1.45 & .35 & 1.46 \\
\hline 12 & .05 & 1.02 & .06 & 1.01 & .04 & 1.75 & .02 & 1.81 \\
\hline 13 & .06 & 1.03 & .07 & 1.03 & .02 & 1.85 & .03 & 1.88 \\
\hline 14 & .04 & 1.01 & .07 & .92 & 0.00 & 3.31 & 0.00 & 3.19 \\
\hline 15 & .03 & 1.01 & .07 & .91 & 0.00 & 5.00 & 0.00 & 4.77 \\
\hline 16 & .03 & 1.00 & .07 & .96 & .96 & 1.00 & .96 & .97 \\
\hline 17 & .03 & 1.02 & .05 & .95 & .24 & 1.55 & .31 & 1.48 \\
\hline 18 & .04 & 1.00 & .06 & .95 & 0.00 & 1.98 & .01 & 1.92 \\
\hline 19 & .03 & .96 & .05 & .96 & .40 & 1.44 & .50 & 1.41 \\
\hline 20 & .07 & .93 & .06 & .95 & .03 & 1.88 & .03 & 1.83 \\
\hline 21 & .08 & .96 & .06 & .95 & .01 & 2.26 & 0.00 & 2.18 \\
\hline 22 & .13 & .90 & .06 & .93 & .68 & .79 & .65 & .79 \\
\hline 23 & .10 & .89 & .07 & .93 & .93 & .95 & .93 & .96 \\
\hline 24 & .13 & .89 & .08 & .93 & .94 & 1.06 & .94 & 1.07 \\
\hline 25 & .10 & 1.16 & .08 & 1.03 & .02 & 2.11 & .02 & 2.05 \\
\hline 26 & .06 & 1.12 & .03 & 1.02 & 0.00 & 3.43 & 0.00 & 3.26 \\
\hline 27 & .04 & 1.12 & .06 & 1.02 & .06 & 4.75 & 0.00 & 4.52 \\
\hline 28 & .05 & 1.01 & .05 & .98 & - & - & - & - \\
\hline 29 & .05 & .97 & .04 & .96 & .63 & 1.31 & .69 & 1.31 \\
\hline 30 & .08 & .95 & .05 & .94 & .21 & 1.53 & .29 & 1.55 \\
\hline 31 & .19 & 1.29 & .06 & 1.09 & 0.00 & 2.79 & 0.00 & 2.56 \\
\hline 32 & .14 & 1.30 & .04 & 1.06 & 0.00 & 5.04 & 0.00 & 4.68 \\
\hline 33 & .16 & 1.30 & .03 & 1.08 & 0.00 & 7.80 & 0.00 & 7.19 \\
\hline 34 & .06 & 1.06 & .05 & .99 & .91 & 1.11 & .93 & 1.09 \\
\hline 35 & .04 & 1.01 & .05 & .96 & .29 & 1.58 & .35 & 1.53 \\
\hline 36 & .07 & .95 & .06 & .94 & .07 & 1.81 & .05 & 1.84 \\
\hline
\end{tabular}

For the $\mathrm{A} \times \theta_{\mathrm{d} i<}$ interaction (see Table 5), $\alpha_{\mathrm{MH}}$ was underestimated for $\theta_{\mathrm{dis}}=0.0$ and $a=$ $1.5(.95)$, and for $\theta_{\text {dis }}=-1.0$ and $a=.5(.94)$. Table 2 shows the corresponding cases for these values of $\theta_{\text {dis }}$ and $a$ across levels of $c$ and $b$ (Cases 13-18 and Cases 19-24). For the $C \times$ $\theta_{\text {dis }}$ interaction, $\alpha_{\mathrm{MH}}$ also was underestimated for $\theta_{\mathrm{dis}}=-1.0$ and $c=.2(.95)$. Again, Table 2 shows the corresponding cases for $\theta_{\mathrm{dis}}=-1.0$ and $c=.2$ across levels of $a$ and $b$ (Cases 22-24, 28-30, and 34-36). Generally, significant effects on $\alpha_{\mathrm{MH}}$ were smaller for the 40 -item test than for the 20 -item test.

A paired $t$ test for cell means between the 20- 
Table 3

Summary of Significant ANOVA Effects

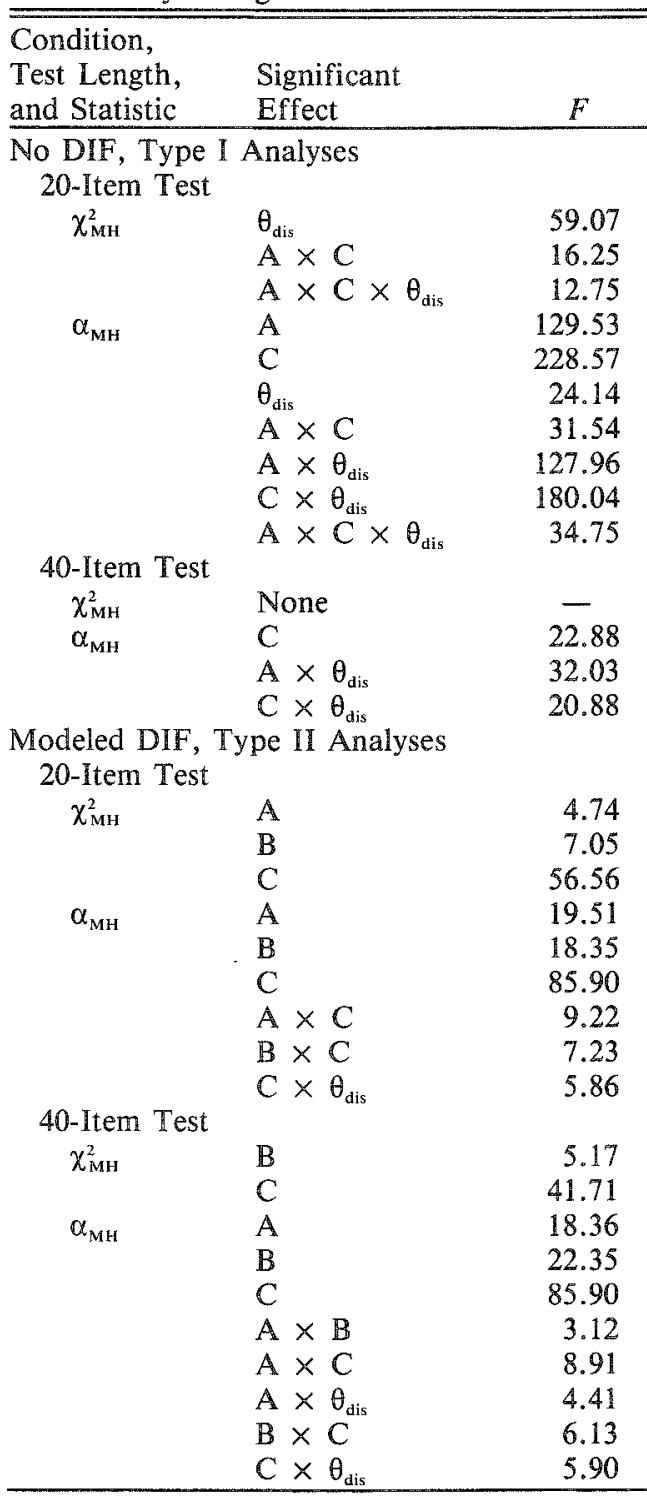

and 40-item tests for the no-DIF conditions was not significant for $\chi_{\mathrm{MH}}^{2}$ [degrees of freedom $(d f)=35, t=1.32, p=.20]$. Means were .06 for the 20 -item test, and .05 for the 40 -item test. The corresponding paired $t$ test was significant for $\alpha_{\mathrm{MH}}(d f=35, t=3.25, p<01)$. Means were 1.03 for the 20 -item test, and .99 for the 40 -item test. Note that although it was statis-
Table 4

$\chi_{\mathrm{MH}}^{2}$ Type I Error Rates and Mean $\alpha_{\mathrm{MH}}$ Values for the $\mathrm{A} \times \mathrm{C} \times \theta_{\text {dis }}$ Interaction in the 20-Item Test, No-DIF Condition

\begin{tabular}{|c|c|c|c|c|}
\hline \multirow{2}{*}{$\begin{array}{l}\text { Statistic } \\
\text { and Value } \\
\text { of } a\end{array}$} & \multicolumn{2}{|c|}{$\theta_{\text {dis }}=0.0$} & \multicolumn{2}{|c|}{$\theta_{\mathrm{dis}}=-1.0$} \\
\hline & $c=0.0$ & $c=.2$ & $c=0.0$ & $c=$ \\
\hline \multicolumn{5}{|l|}{$\chi_{\mathrm{MH}}^{2}$} \\
\hline$a$ & $\begin{array}{l}.04 \\
.04\end{array}$ & & $\begin{array}{l}.06 \\
.06\end{array}$ & .06 \\
\hline$a=$ & .04 & .0 & .16 & .05 \\
\hline \multicolumn{5}{|l|}{$\alpha_{\mathrm{MH}}$} \\
\hline$a$ & 1.00 & 1. & 9 & .89 \\
\hline$a=1.0$ & 1.00 & 1.00 & 1.13 & .98 \\
\hline$a=1.5$ & 1.01 & 1.01 & 1.30 & 1.00 \\
\hline
\end{tabular}

tically significant, the overall effect of test length on $\alpha_{\mathrm{MH}}$ was small.

\section{DIF Analyses}

Results for the DIF analyses are presented in two stages: first in terms of the ANOVA analyses based on focal group IRFs, with special attention given to parameter $\times \theta_{\text {dis }}$ interactions, and second in terms of form and degree of DIF.

20-item test. ANOVAs for the $\chi_{M H}^{2}$ Type II conditions produced significance for the $\mathrm{A}, \mathrm{B}$, and $\mathrm{C}$ main effects. Means were .42 for $a=.5$, .14 for $a=1.0$, and .21 for $a=1.5 ; .41$ for $b=0.0, .27$ for $b=.3$, and .14 for $b=.5$; and .04 for $c=0.0$ and .46 for $c=.2$. The ANOVA results for $\alpha_{M H}$ for the 20 -item test in the DIF condition indicated significance for the $\mathrm{A}, \mathrm{B}$, and $\mathrm{C}$ main effects and the $\mathrm{A} \times \mathrm{C}, \mathrm{B} \times \mathrm{C}$, and $\mathbf{C} \times \theta_{\text {dis }}$ interactions. Main effect means were

Table 5

$A \times \theta_{\text {dis }}$ and $\mathrm{C} \times \theta_{\text {dis }}$ Interactions

for $\alpha_{M H}$ Type I Cases From the 40-Item Test in the No-DIF Condition

\begin{tabular}{lcc}
\hline \hline Interaction and & & \\
Parameter Value & $\theta_{\text {dis }}=0.0$ & $\theta_{\text {dis }}=-1.0$ \\
\hline $\mathrm{A} \times \theta_{\text {dis }}$ & & \\
$a=.5$ & 1.01 & .94 \\
$a=1.0$ & 1.02 & .99 \\
$a=1.5$ & .95 & 1.02 \\
$\mathrm{C} \times \theta_{\text {dis }}$ & & \\
$c=0.0$ & 1.00 & 1.02 \\
$c=.2$ & 1.00 & .95 \\
\hline
\end{tabular}


$1.59,2.49$, and 2.75 for $A ; 1.56,2.23$, and 3.00 for $\mathbb{B}$; and 3.15 and $\mathbb{1} .19$ for $C$. Table 6 shows the means for the $C \times \theta_{\mathrm{dis}}$ interaction.

Table 6

$\mathrm{C} \times \theta_{\text {dis }}$ Interaction for $\alpha_{\mathrm{MH}}$ Type II Cases for the 20-Item Test in the DIF Condition

\begin{tabular}{ccc}
\hline$c$ & $\theta_{\text {dis }}=0.0$ & $\theta_{\text {dis }}=-1.0$ \\
\hline$c=0.0$ & 2.80 & 3.50 \\
$c=.2$ & 1.43 & 1.27 \\
\hline
\end{tabular}

When $c=0.0$ in the focal group, $\theta_{\text {dis }}=-1.0$ raised $\alpha_{\mathrm{MH}}$. Because $c=.2$ in the reference group, there was considerable DIF at lower $\theta$ levels that was weighted by the distribution of focal group examinees.

40-item iest. The ANOVA for $\chi_{M M H}^{2}$ for the 40-item test in the DIF condition produced significance for the $\mathbb{B}$ and $\mathbb{C}$ main effects. Means were $.41, .28$, and .13 for $B$, and .04 and .52 for C. The ANOVA for $\alpha_{\mathrm{MH}}$ for the 40 -item test in the DIF condition yielded significance for the $\mathbb{A}, \mathbb{B}$, and $\mathrm{C}$ main effects and the $\mathrm{A} \times \mathrm{B}, \mathrm{A} \times \mathrm{C}$, $A \times \theta_{\text {dis }}, B \times C$, and $C \times \theta_{\text {dis }}$ interactions. Means were $1.55,2.47$, and 2.75 for $A ; 1.52,2.17$, and 2.92 for $\mathbb{B}$; and 3.05 and 1.19 for $C$. The $A \times \theta_{\text {dis }}$ and $C \times \theta_{\text {dis }}$ interactions are shown in Table 7 .

Tabe 7

A $\times \theta_{\text {dis }}$ and $\mathrm{C} \times \theta_{\text {dis }}$ Interactions: $\alpha_{\mathrm{MH}}$ Type II Cases for the 40-1tem Test in the DIF Condition

\begin{tabular}{lcc}
\hline Interaction and & & \\
Parameter Value & $\theta_{\text {dis }}=0.0$ & $\theta_{\text {dis }}=-1.0$ \\
\hline $\mathrm{A} \times \theta_{\text {dis }}$ & & \\
$a=.5$ & 1.72 & 1.37 \\
$a=1.0$ & 2.40 & 2.54 \\
$a=1.5$ & 2.37 & 3.14 \\
$\mathrm{C} \times \theta_{\text {dis }}$ & & \\
$c=0.0$ & 2.79 & 3.29 \\
$c=.2$ & 1.42 & 1.27 \\
\hline
\end{tabular}

For the $\mathrm{A} \times \theta_{\text {dis }}$ interaction, Table 7 indicates that, compared with the corresponding $\theta_{\text {dis }}=0.0$ $\alpha_{\mathrm{MH}}$ levels, $\theta_{\mathrm{dis}}=-1.0$ resulted in a lower $\alpha_{\mathrm{MH}}$ for $a=.5$ and a higher $\alpha_{\mathrm{MH}}$ for $a=1.5 . a=1.0$ values were nearly equivalent intermediate values in a disordinal interaction. The interaction was significant and the values reported show the trend underlying the interaction. For the $\mathrm{C} \times \theta_{\mathrm{dis}}$ interaction, the values in Table 7 are similar to those in Table 6. However, the increase of $\alpha_{\mathrm{MH}}$ for the $c=0.0, \theta_{\mathrm{dis}}=-1.0$ condition was slightly smaller for the 40 -item test than the 20 -item test $\left(\alpha_{\mathrm{MH}}\right.$ increased from 2.80 to 3.50 for the 20 -item test and from 2.79 to 3.29 for the 40-item test).

A paired $t$ test for the overall cell means between the 20- and 40-item tests for the DIF conditions was not significant for $\chi_{M H}^{2}(d f=33$, $t=-.19, p=.85$ ). Means were .27 for the 20 and 40 -item tests. The corresponding paired $t$ test was significant for $\alpha_{\mathrm{MH}}(d f=33, t=2.39$, $p=.02)$. Means were 2.30 for the 20-item test, and 2.25 for the 40 -item test. Note that although it was statistically significant, the overall effect of test length on $\alpha_{M H}$ was small, as for the noDIF condition.

\section{Fon and Degree of D D}

The values of CSA and CUA calculated using formulas presented in $\operatorname{Kim} \&$ Cohen (1991) are presented in Table 8. These levels of DIF are consistent with DIF modeled in other studies but include an expanded range of high DIF items, due to the comprehensive manipulation of IRF parameters. Donoghue et al. (1993) investigated relatively low levels of DIF in the range $.39 \leq \mathrm{CSA} \leq .48$; Swaminathan \& Rogers (1990) investigated the two quantities CUA $=.60$ and $\mathrm{CUA}=.80$ for uniform and nonuniform conditions. Hambleton \& Rogers (1988), in a study using real data, examined the range of areas $.12 \leq \mathrm{CUA} \leq .95$.

CSA and CUA are equivalent when the DIF is uniform; CSA will be smaller than CUA when the DIF is nonuniform. The relationship between the magnitudes of CSA and CUA indicates the extent of nonuniformity. In the extreme case of symmetric, nonuniform DIF about the intersection point of the IRFS, CSA $=0$ for any value of CUA (e.g., Cases 4 and 22, and Cases 16 and 34, see Tables 1 and 8).

In the present study, $x_{M H}^{2}$ Type $I I$ error rates were considered low if they were .20 or lless, 
Table 8

CSA and CUA Values for Modeled DIF Conditions

\begin{tabular}{lcr}
\hline $\begin{array}{l}\text { Modeled DIF } \\
\text { Case Numbers }\end{array}$ & CSA & CUA \\
\hline 1 and 19 & 1.20 & 1.20 \\
2 and 20 & 1.76 & .76 \\
3 and 21 & 2.13 & .13 \\
4 and 22 & 0.0 & .51 \\
5 and 23 & .44 & .78 \\
6 and 24 & .74 & .99 \\
7 and 25 & 1.20 & 1.20 \\
8 and 26 & 1.80 & 1.80 \\
9 and 27 & 2.19 & 2.19 \\
10 and 28 & - & - \\
11 and 29 & .48 & .48 \\
12 and 30 & .79 & .79 \\
13 and 31 & 1.20 & 1.34 \\
14 and 32 & 1.80 & 1.83 \\
15 and 33 & 2.20 & 2.21 \\
16 and 34 & 0.0 & .21 \\
17 and 35 & .48 & .54 \\
18 and 36 & .80 & .82 \\
\hline
\end{tabular}

corresponding to power levels of .80 or greater. CUAS from Table 8 , and $\chi_{M H}^{2}$ Type II error rates and $\alpha_{M H}$ levels from Table 2, were considered together. Overall effects of test length on $\chi_{\mathrm{MH}}^{2}$ and $\alpha_{\mathrm{MH}}$ also are reported.

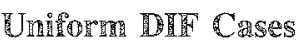

Low $\chi_{M H}^{2}$ Type II error rates were obtained when large DIF was present across most of the $\theta$ scale, for both 20 -and 40-item tests, with or without $\theta$ discrepancy. Examples of such conditions are Cases 2 and 20, 3 and 21,7 and 25,8 and 26, and 9 and 27, which had mean $\alpha_{M H} \geq 1.80$ and CSA $=$ CUA $\geq 1.20$ (see Table 8). A high Type II error rate was obtained for small DIF across the $\theta$ scale, for both test length and $\theta$ discrepancy conditions. For example, for Cases 11 and $29 \alpha_{\mathrm{MH}}=1.45$ and 1.31 , respectively, and $\mathrm{CSA}=\mathrm{CUA}=.48$.

For certain uniform DIF cases with low Type II error rates under the no-discrepancy $\theta$ condition, high Type II error rates were obtained under the discrepant $\theta$ condition, for both 20 and 40-item tests. Examples include Cases 1 and 19 and Cases 12 and 30 , which had $1.41 \leq \alpha_{\mathrm{MH}}$ $\leq 1.81$ and $.79 \leq \mathrm{CSA}=\mathrm{CUA} \leq 1.20$. The $\theta$ discrepancy weights segments between the reference and focal IRFs having small areas, that is, where the IRFs are close, even though overall DIF is substantial.

\section{Nonuminionim lon Cases}

High Type II error rates were obtained when there was nonuniformity across the $\theta$ range, that is, when the IRFs crossed at or near $\theta=0.0$, for discrepant and nondiscrepant $\theta$ distributions and for 20- and 40-item tests. Cases include Cases 4 and 22, Cases 5 and 23, Cases 6 and 24, Cases 16 and 34, and Cases 17 and 35, which had $.79 \leq \alpha_{\mathrm{MH}} \leq 1.58,0.0 \leq \mathrm{CSA} \leq .74$, and $.21 \leq$ CUA $\leq .99$, representing a range of values from low to moderately high levels of DIF.

Low Type II error rates were obtained when nonuniformity was small compared to overall DIF across the range of $\theta$, for both $\theta$ conditions and test lengths. Examples include Cases 13 and 31 , Cases 14 and 32, Cases 15 and 22, and Cases 18 and 36 , which had $1.85 \leq \alpha_{M H} \leq 7.80$, $.80 \leq \mathrm{CSA} \leq 1.20$, and $.82 \leq \mathrm{CUA} \leq \mathbb{1 . 3 4}$. The procedure overcame a small degree of nonuniformity in the upper end of the $\theta$ scale due to the large degree of overall DIF for these cases.

\section{Discression}

The simulations reported here studied the empirical $\chi^{2}$ Type I and Type $\mathbb{I}$ error rates and estimated odds-ratios for the MH procedure under a variety of IRT parameterizations. Results were studied for both 20 -item and 40-item tests. Values of the $a, b$, and $c$ parameters for the studied item were varied systematically. Parameterizations for the remaining items varied across items but included items with 2PLM and 3PLM IRFS. Under this condition, the total test score used for matching in the $\mathrm{MH}$ procedure was not a sufficient statistic for the latent trait parameter. Because all simulations were performed within the IRT framework, the conclusions that follow are limited to conditions approaching those modeled here.

For the no-DIF conditions, test length 
strongly influenced the Type $\mathbb{I}$ error rates and $\alpha_{\mathrm{MH}}$ estimates. For the 20 -item test, the $\mathrm{MH}$ procedure gave inflated error rates and misestimated $\alpha_{\mathrm{MH}}$ values under many conditions when the groups differed in prior $\theta$ distributions. The values of both the $a$ and $c$ parameters influenced these results. These results are consistent with theoretical predictions that suggest that under multiparameter models Type I errors will occur when the group $\theta$ distributions are ordered, as found here (Meredith \& Millsap, 1992; Zwick, 1990). They also augment the findings of Donoghue et al. (1993), who found inflation in $\alpha_{\mathrm{MH}}$ under a limited set of no-DIF conditions, and Swaminathan \& Rogers (1990) who studied some multiparameter cases, but did so only for long tests. The inflation in the Type I error rate disappeared entirely in the 40-item test, although the $\alpha_{\mathrm{MH}} \mathrm{s}$ continued to be influenced, to some extent, by studied factors. This suggests the importance of evaluating $\alpha_{\mathrm{MH}}$ and $\chi_{\mathrm{MH}}^{2}$ jointly. Overall, the 40 -item test length appeared to be adequate for "near-sufficiency" to hold under the studied conditions, as described by Meredith \& Millsap (1992).

For the DIF conditions, ANOVA results indicated that certain parameter $\times \theta$ level interactions influenced the level of $\alpha_{\mathrm{MH}}$ for both 20- and 40 -item tests. Interactions involving $\theta$ distributions did not, however, influence the performance of $\chi_{M H}^{2}$. This again suggests the importance of interpreting $\alpha_{\mathrm{MH}}$ along with the $\chi_{\mathrm{MH}}^{2}$ significance test under $\theta$ discrepant conditions. No conclusions were drawn from the ANOVA analyses for DIF conditions with regard to parameter main effects or interactions because these were deliberately varied to create DIF.

Instead, the influence of form and degree of DIF on the results was considered. Large uniform DIF was readily detected, with one exception. The exception to this pattern was Case 19 in which the focal group parameterization was $a=.5$, $b=0.0$, and $c=0.0$. In this case, the largest IRF differences occurred in regions of the $\theta$ scale in which there was little overlap between groups in their $\theta$ distributions. This fact appears to explain the low power. Small uniform DIF, as modeled in Cases 11 and $29(\mathrm{CSA}=\mathrm{CUA}=.48)$, was not readily detected by the $\mathrm{MH}$ procedure.

In the nonuniform DIF case, the MH procedure was often unable to detect DIF regardless of the relative positions of the group $\theta$ distributions. High Type II error rates were recorded even when the amount of DIF was relatively large (CUA $=.99)$, and $\alpha_{M H}$ estimates were low in these cases. Note that the amount of DIF studied here was larger in many cases than that studied in other simulation studies (Donoghue et al., 1993; Swaminathan \& Rogers, 1990). The results found here are consistent with those of Swaminathan \& Rogers (1990; Rogers \& Swaminathan, 1990), and suggest that the MH procedure generally has low power to detect nonuniform DIF. However, when the degree of nonuniformity is small relative to the amount of DIF (e.g., Cases 14 and 32) the procedure may have adequate power. The results also indicated that test length generally had little effect on Type II error rates and $\alpha_{\mathrm{MH}} \mathrm{s}$ under DIF conditions.

The results can be reduced to four broad conclusions. First, under no-DIF conditions the $\mathrm{MH}$ procedure will falsely indicate DIF (considering both $\alpha_{\mathrm{MH}}$ and $\chi_{\mathrm{MH}}^{2}$ ) in short tests (less than 20 items, based on the observed trends for 40- and 20-item tests), when the items fit multiparameter IRT models and groups differ on $\theta$ level. This problem seems to disappear in long tests ( $\geq 40$ items). Secondly, under DIF conditions these interactions influence $\alpha_{\mathrm{MH}}$, but not $\chi_{\mathrm{MH}}^{2}$, for both test lengths. This provides support for using both $\alpha_{\mathrm{MH}}$ and $\chi_{\mathrm{MH}}^{2}$ information when evaluating DIF. Third, the $\mathrm{MH}$ procedure can readily detect large uniform DIF that is present in regions of the $\theta$ scale in which most examinees are found. Fourth, the procedure has low power to detect nonuniform DIF even if the amount of DIF is substantial. There may be exceptions to this if the degree of nonuniformity is small compared to the overall amount of DIF.

There are potential influences on MH performance beyond those studied here. Sample size was not varied in the simulations. In small sam- 
ples, lower power is expected for the $\chi_{M H}^{2}$ test. Swaminathan \& Rogers (1990) confirmed this prediction for samples of size 250. The influence of sample size on $\alpha_{M H}$ is less clear. Zieky (1993) recommended that the sample size for operational use of the MH procedure should be at least 500 for the reference and focal groups combined. A second possible influence on $\mathrm{MH}$ performance is the amount of DIF in items other than the studied item. The results given here concerned the ideal case in which DIF is present only in the studied item. Donoghue et al. (1993) studied the influence of biased items in the matching variable, but only under uniform bias conditions. Rogers \& Swaminathan (1990) varied the percentage of biased items $(0 \%$ versus $15 \%)$ in their simulations and found small effects for this variable. One mitigating factor in their simulations may have been the long test length used, which resulted in many unbiased items in the test even in the $15 \%$ condition. More research is needed to fully explore the influence of biased items in the matching criterion on $\mathrm{MH}$ performance.

\section{References}

Donoghue, J., Holland, P. W., \& Thayer, D. T. (1993). A Monte Carlo study of factors that affect the Mantel-Haenszel and standardization measures of differential item functioning. In $\mathrm{P} . \mathrm{W}$. Holland $\& \mathrm{H}$. Wainer (Eds.), Differential item functioning ( $\mathrm{pp}$. 137-166) Hillsdale NJ: Erlbaum.

Hambleton, R. K., \& Rogers, H. J. (1988). Detecting potentially biased test items: Comparison of IRT area and Mantel-Haenszel methods. Applied Measurement in Education, 2, 313-334.

Holland, P. W. \& Thayer, D. T. (1988). Differential item performance and the Mantel Haenszel procedure. In H. Wainer \& H. I. Braun (Eds.), Test validity (pp. 129-145). Hillsdale NJ: Erlbaum.

Kim, S. \& Cohen, A. S. (1991). A comparison of two area measures for detecting differential item functioning. Applied Psychological Measurement, 15, 269-278.

Lewis, C. (1993). A note on the value of including the studied item in the test score when analyzing test items for DIF. In P. W. Holland \& H. Wainer (Eds.), Differential item functioning (pp. 317-319). Hillsdale NJ: Erlbaum.

Lord, F. M. (1980). Applications of item response theory to practical testing problems. Hillsdale $\mathrm{NJ}$ : Erlbaum.

Mantel, N., \& Haenszel, W. (1959). Statistical aspects of the analysis of data from retrospective studies of disease. Journal of the National Cancer Institute, 22, 719-740.

Meredith, W., \& Millsap, R. E。 (1992). On the misuse of manifest variables in the detection of measurement bias. Psychometrika, 57, 289-311.

Millsap, R.E., \& Meredith, W. (1992). Inferential conditions in the statistical detection of measurement bias. Applied Psychological Measurement, 16 , 389-402.

Rogers, H. J., \& Swaminathan, H. (1990, April). A comparison of the logistic regression and MantelHaenszel procedures for detecting differential item functioning. Paper presented at the annual meeting of the American Educational Research Association, Boston.

Swaminathan, H., \& Rogers, H. J. (1990). Detecting differential item functioning using logistic regression procedures. Journal of Educational Measurement, 27, 361-370.

Zieky, M. (1993). Practical questions in the use of DIF statistics in test development. In P. W. Holland \& $\mathrm{H}$. Wainer (Eds.), Differential item functioning ( $\mathrm{pp}$. 337-347). Hillsdale NJ: Erlbaum.

Zwick, R. (1990). When do item response function and Mantel-Haenszel definitions of differential item functioning coincide? Journal of Educational Statistics, $15,185-197$.

\section{Acknow}

Preparation of this article was supported in part by grant HMH16242 NIMH, Rutgers Center for Research on the Organization and Financing of Care for the Severely Mentally Ill. The authors thank James Uttaro for computing assistance and three anonymous reviewers for comments on previous drafis of this article.

\section{Author's Address}

Send requests for reprints or further information to Thomas Uttaro, Rutgers University, 30 College Avenue, New Brunswick NJ 08903, U.S.A. Internet: teugc@cunyvm.cuny.edu. 